\title{
Las remesas y su incidencia en el nivel de producción a corto plazo
}

Richard Roca Garay

\section{RESUMEN}

El artículo analiza los posibles efectos de las remesas sobre el nivel de producción interno bajo tipo de cambio fijo o flexible, con alta movilidad de capitales, y encuentra que tendría un efecto expansivo si se mantiene el tipo de cambio y contractivo si deja libre el tipo de cambio.

Palabras clave: Macroeconomía abierta, movilidad de capitales, remesas de divisas, tasa de interés, política monetaria.

\section{ABSTRACT}

The article discusses the potential impact of remittances on the level of domestic output under fixed exchange rate and flexible with high capital mobility and finds that it would have an expansionary effect if the exchange rate and contractive if the exchange rate is free.

Keywords: Open Macroeconomics, capital mobility, remittances, interest rate, monetary policy. 


\section{Richard Roca Garay}

\section{INTRODUCCIÓN}

Este artículo analiza, bajo el enfoque flujo del mercado libre de divisas, la forma como las remesas de dólares de los peruanos en el exterior a sus familiares del país tiende a afectar al nivel de actividad del país.

Los diversos textos de macroeconomía internacional como los de Krugman, Salvatore, Appleyard, Obstfeld y Rogoff, Tugores, entre otros, omiten el análisis de la influencia de las remesas sobre el nivel de actividad de una economía abierta. Este trabajo desarrolla un modelo de tipo IS-LM abierta de corto plazo en el que se incluye la interdependencia de los mercados de divisas con la balanza de pagos, el mercado de activos financieros y el mercado de bienes, en un contexto de alta movilidad de capitales pero no perfecta, tanto para tipo de cambio fijo como para tipo de cambio flexible.

Se encuentra que a corto plazo con tipo de cambio fijo el efecto es expansivo; y con tipo de cambio libre, el efecto es contractivo

\section{EL MODELO}

Seguidamente, mostramos un modelo en el marco de la IS-LM para una economía abierta con alta movilidad de capitales pero no perfecta de corto plazo, en el cual se considera la influencia mutua de los mercados de divisas a través de la balaza de pagos, los mercados financieros y de dinero y el mercado de bienes.

\subsection{El Mercado de Bienes}

La condición de equilibrio del mercado de bienes nacionales se resume en la siguiente ecuación en la que la Producción Interna Bruta $(Y)$ se iguala a la Demanda Agregada $(D A)$, la cual está conformada por el Consumo $(C)$, la Inversión $(I)$ el Gasto de Gobierno $(G)$ y las Exportaciones Netas $(X N)$ :

$$
Y=C\left(D_{+}\right)+I(\bar{r})+\bar{G}+\boldsymbol{X}\left(\frac{\underline{e}^{*}}{P_{+}}, Y_{+}^{*}, Y\right)
$$




\section{Las remesas y su incidencia en el nivel de producción a corto plazo}

El consumo depende directamente del Ingreso Disponible (YD), al cual definimos como igual a la producción interna menos la recaudación tributaria más las donaciones que se reciben del exterior:

$$
\boldsymbol{D}=Y-\tau \cdot Y+\overline{B T R}
$$

Donde $\tau \cdot Y$ es una recaudación tributaria que depende del nivel de la renta, $\overline{B T R}$ son las donaciones internacionales que se reciben del exterior en forma de remesas. La inversión depende inversamente de la tasa de interés real $(r)$, el gasto de gobierno se asume que es fijado exógenamente por el gobierno, y las exportaciones netas dependen directamente del tipo de cambio nominal $(E)$ y del nivel de precios externo $\left(P^{*}\right)$, e inversamente del nivel de precios nacional $(P)$, del nivel de producción del resto del mundo $\left(Y^{*}\right)$ e inversamente del nivel de producción nacional $(Y)$.

Diferenciando totalmente:

$$
\begin{aligned}
& \ddot{d}=C_{\Downarrow}(1-\tau) \bar{d}-Y \cdot C_{\Downarrow} d \tau+C_{\Downarrow} d \overline{B T R}+I_{r} d+d \bar{A}+\boldsymbol{X}_{R} \frac{P^{*}}{P} \mathbb{E}+\boldsymbol{X}_{R} \frac{E}{P} \not \mathbb{P}^{*} \\
& -X_{R} \frac{E^{*}}{P^{2}} \not P+X_{Y^{*}} \mathbb{Z}^{*}+X_{Y} \not
\end{aligned}
$$

Donde $\bar{A}$ represente la suma de los diferentes componentes de la demanda agregada interna autónoma.

\subsection{El Mercado de dinero y sistema financiero nacional}

Considerando que el Banco Central puede emitir dinero $(H)$ mediante la compra de valores financieros (VF) o prestando a agentes internos (CIN) o que puede comprar activos financieros internacionales $(R I N)$ como dólares:

$$
\underset{+}{L(Y, i)}=\frac{H}{P}=\frac{F+C I N+R I N}{P}
$$

Diferenciando totalmente:

$$
L_{Y} \boldsymbol{d}+L_{i} \dot{\boldsymbol{d}}=\frac{d V F+d C I N+d R I N}{P}-\frac{H}{P^{2}} \not P
$$




\section{Richard Roca Garay}

\subsection{El Sector Externo}

El enfoque flujo del mercado de divisas afirma que el equilibrio de los movimientos autónomos de la balanza de pagos $(B P)$ implica el equilibrio del mercado de divisas. Cuando el saldo de la balanza de pagos es cero, la oferta y demanda de divisas se igualan:

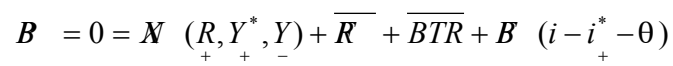

Donde $\mathrm{XN}$ son las exportaciones netas, $R F$ la renta de factores que suponemos exógena, y BF la entrada neta de capitales, la cual depende directamente del diferencial de rendimientos a favor de los activos nacionales.

Diferenciando totalmente:

$$
\begin{aligned}
& 0=\mathbb{N}_{R} \frac{P^{*}}{P} \mathbb{E}+\boldsymbol{X}_{R} \frac{E}{P} \mathbb{P}^{*}-\boldsymbol{X}_{R} \frac{E^{*}}{P^{2}} \not \mathbb{P}+\boldsymbol{X}_{Y^{*}} \boldsymbol{d}^{*}+\boldsymbol{X}_{Y} \boldsymbol{Z} \\
& +d \bar{R}+d \overline{B T R}+B_{(\cdot)} \dot{d}-B_{(.)} \dot{d}^{*}-B_{(.)} d \theta
\end{aligned}
$$

\section{TIPO DE CAMBIO FIJO}

Con tipo de cambio fijo, la oferta monetaria $(H)$ es endógena; y el tipo de cambio nominal $(E)$, exógeno.

De (1.1), (2.1) y (3.1) si solo cambia la variable exógena Balanza de Transferencias $(B T R)$ se obtiene la forma reducida de las modificaciones del nivel de producción de equilibrio de corto plazo:

$$
\frac{d}{d \overline{B T R}}=\frac{C_{\text {и }}-\frac{I_{r}}{B_{(\cdot)}}}{S_{Y}-\left(1-\frac{I_{r}}{B_{(\cdot)}}\right) X_{Y}}>0
$$

Lo cual señala que una mayor entrada de divisas por remesas provenientes del exterior eleva el ingreso disponible y lo cual genera presiones a la baja al tipo de cambio; pero ello obliga al Banco Central a comprar divisas para que se mantenga el tipo de cambio por lo que aumenta endógenamente la oferta monetaria reforzando el efecto expansivo. 


\section{Las remesas y su incidencia en el nivel de producción a corto plazo}

\section{TIPO DE CAMBIO FLEXIBLE}

Con tipo de cambio flexible, la oferta monetaria $H$ es exógena y el tipo de cambio endógeno.

De (1.1), (2.1) y (3.1) se obtiene la forma reducida de las modificaciones del nivel de producción de equilibrio de corto plazo:

$$
\frac{d}{d \overline{B T R}}=-\frac{-\left(1-C_{\sharp}\right)}{S_{Y}-\frac{\left(B_{(\cdot)}-I_{r}\right)_{Y}}{L_{i}}}<0
$$

Lo cual señala que una mayor entrada de divisas por remesas provenientes del exterior tiene un efecto neto negativo sobre el nivel de producción de equilibrio de corto plazo. Si bien al incrementarse las remesas al país aumenta el ingreso disponible, dicha entrada de divisas aumenta la oferta de divisas y deteriora las exportaciones netas, lo cual es más fuerte que el efecto sobre el consumo por lo que, en neto, cae el nivel de producción a corto plazo.

Esto nos lleva postular que el Perú, en donde hay un tipo de cambio flexible aunque con flotación sucia, el efecto expansivo se haya debido al aumento de las compras de divisas de parte del BCRP, lo cual incrementó la oferta monetaria de forma muy sensible provocando un efecto neto expansivo.

\section{CONCLUSIONES}

Con tipo de cambio fijo la mayor entrada de remesas, tendría efecto expansivo. Con tipo de cambio flexible, tendría efecto recesivo - a menos que el BCRP no permita una caída fuerte del tipo de cambio, lo que implica que recurrentemente compre divisas aumentando la cantidad de dinero, que es lo que habría estado sucediendo en el Perú-.

\section{BIBLIOGRAFÍA}

Appleyard, Daniel y Field, A (2003). International Economics. McGraw-Hill.

Blanchard, Olivier (2004). Macroeconomics. Pearson. 


\section{Richard Roca Garay}

Hustad y Melvin (2007). International Economics. 7. ${ }^{\text {a }}$ ed., Pearson.

Krugman, Paul y Maurice Obtsfeld (2005). International Economics. McGraw-Hill.

Obstfeld, Maurice y Maurice Rogoff (1996) International Macroeconomics. MIT Press.

Salvatore, Dominick (1995). Economía Internacional. McGraw-Hill.

Tugores, J. (1994). Economía Internacional de Integración Económica. McGraw-Hill. 